Send your letters to the Editor,

British Dental Journal,

64 Wimpole Street

London

W1G 8YS

E-mail bdj@bda.org

Priority will be given to letters less than 500 words long.

Authors must sign the letter, which

may be edited for reasons of space.

\section{NO PROBLEM UP NORTH}

Sir, in articles published on pages 285 and 288 in the news section of the $B D J$ (2008; 204) Teresa Perchard, Policy Director of the Citizens Advice Bureau, is reported as saying in her evidence to the Health Select Committee that that the South West and North West were 'dental deserts' where access is particularly poor.

The North West does not have a significant dental access problem. In fact using the Department of Health's measure of access which is the number of individuals who have attended during the last 24 months, access in the North West is better than the national average. Latest figures available from the Information Centre (June 2007) show that $60 \%$ of the population in the North West had visited the dentist in the last two years compared to 55\% for England as a whole. Access in the North West has been remarkably constant over the last two years or so, even though we lost about $5 \%$ of dentists when the new dental contract was introduced in April 2006. So I am puzzled why the CAB has identified the North West as having a severe problem.

The North West Strategic Health Authority recognises that improvements are needed and there are a small number of areas where access to NHS dental services is a problem. In March 2007 the SHA asked all of the PCTs in the North West to produce a local oral health strategy to set out how they will improve the dental health of their population and increase access to dental services. These plans are now being implemented and many PCTs have made significant investments in dental services from their general allocations over and above the recently announced 11\% uplift in ring-fended funding from the Department of Health.

I think there is a large gap between public and media perceptions of obtaining access to NHS dental services and the real situation. The efforts made by the NHS to shrink this gap and help members of the public to access dental services is not helped by the inaccurate and misleading statements attributed to Ms Perchard.

M. Tickle

Consultant in Dental Public Health and Dental lead for the North West

Strategic Health Authority

DOI: 10.1038/sj.bdj.2008.509

\section{UNSUITABLE CURRENCY}

Sir, I write in support of the letter from Dr Alex Crawford (BDJ 2008; 204: 629) underlying the importance of sedation in preventing phobia in dentistry. Dr Crawford was focussing on inhalation sedation but I believe his point is also valid for IV sedation. The DH report in $2003^{1}$ (p 20) states that the prevention of phobia is a clear indication for the use of conscious sedation.

Unfortunately, once a patient has become phobic the use of standard sedation techniques may not be successful. A consultant anaesthetist once referred to this as the 'midazolam bouncing off the patient'. Most practitioners of IV sedation using midazolam will understand this comment. In this situation, more advanced sedation techniques are often required. In some cases a GA will be necessary. This is precisely what we are trying to avoid.

The UDA is not a suitable currency for the dental treatment of high needs patients. It is even more unsuitable for patients who require sedation. The new contract has restricted the availability of sedation in the NHS. The practitioner is credited with no UDAs for sedation. Furthermore, in my experience, a referral to a nearby teaching hospital produces a standard letter which states that: 'This hospital does not offer sedation for routine restorative treatment' and the return of the referral letter.

This means that more phobic patients may be produced. Such patients often require extensive treatment and their oral health deteriorates as they cannot access the appropriate care. Attempts to treat without sedation exacerbate the situation and make a GA more likely.

The only way forward for sedation in NHS dentistry is to use a more suitable currency than the UDA.

M. Barratt Sale

1. Standing Dental Advisory Committee, Department of Health. Conscious sedation in the provision of dental care: report of an expert group on sedation for dentistry. London: Department of Health, 2003.

DOI: 10.1038/sj.bdj.2008.510

\section{CONTINUING CONTROVERSY}

Sir, the NICE guidance does not recommend any antibiotic prophylaxis, even for dental procedures in patients with high risk cardiac lesions ${ }^{1}$ and correspondence from Martin clearly supports this approach. ${ }^{2}$ However, the American Heart Association (AHA) reviewed similar scientific literature to that considered by NICE and does recommend prophylaxis for high risk cardiac patients. ${ }^{3}$

Martin is correct to state that endocarditis is unlikely to follow a single dental procedure but this does not necessarily mean there is no risk. An analogy can be made with hip joint replacement surgery 
where it is unlikely that postoperative deep sepsis will occur, but nonetheless the consequences of such infection may be catastrophic when it does happen. Streptococcal endocarditis in a patient with a prosthetic heart valve, an example of a high risk cardiac condition, may well result in death.

The NICE guidelines, and Martin, refer to tooth brushing as a risk but there is no direct evidence that this has ever caused endocarditis. Also, toothbrushing generally causes significant viridans streptococcal bacteraemia only when severe periodontitis is present. ${ }^{4}$ There is compelling indirect evidence for the importance of dental extractions as both a cause of highly predictable streptococcal bacteraemia and of occasional cases of viridans streptococcal endocarditis. ${ }^{4}$ Extractions, and surgery involving the gums or teeth, continue to warrant antibiotic prophylaxis against endocarditis for patients with high risk cardiac conditions, as recommended by the latest AHA report. ${ }^{3}$

Martin is not correct to state there is no evidence that amoxicillin would be effective for prophylaxis. ${ }^{2}$ It would be more accurate to note that there is no controlled clinical trial data on the efficacy of amoxicillin for preventing endocarditis after dental procedures, partly because of the rarity of the disease. However, there is strong evidence that shows a single dose of amoxicillin can be highly effective in preventing streptococcal endocarditis in a stringent experimental animal model. ${ }^{4}$ There is markedly conflicting data from different bacteraemia studies and some of this may arise from different blood culture methods used. ${ }^{4,5}$

The NICE guidance suggests that the risk of fatal anaphylaxis from amoxicillin prophylaxis is so great that it would result in more deaths from anaphylaxis than the number of endocarditis deaths prevented by giving amoxicillin prophylaxis. However, this fear is probably exaggerated as there have been no reports of deaths from anaphylaxis following amoxicillin prophylaxis against endocarditis, either in America ${ }^{2}$ or the UK. ${ }^{6}$

D. Shanson Hon Consultant Microbiologist
1. National Institute for Health and Clinical Excellence. Prophylaxis against infective endocarditis 2008 (NICE clinical guideline no. 64).

2. Martin M V. An end to antimicrobial prophylaxis against infective endocarditis for dental procedures? Br Dent J 2008; 204: 107.

3. Wilson W, Taubert K, Gewitz M et al. Prevention of infective endocarditis. Guidelines from the American Heart Association Rheumatic Fever, Endocarditis and Kawasaki Disease Committee. Circulation 2007: 116: 1736-1754.

4. Shanson D. New British and American guidelines for the antibiotic prophylaxis of infective endocarditis: do the changes make sense? A critical review. Curr Opin Infect Dis 2008; 21: 191-199.

5. Shanson D. Conflicting advice. Br Dent J 2006; 201: 613-614.

6. Lee P, Shanson D. Results of a UK survey of fatal anaphylaxis after oral amoxicillin. J Antimicrob Chemother 2007; 60: 1172-1173.

DOI: $10.1038 /$ sj.bdj.2008.511

\section{UNDERLYING PROBLEMS}

Sir, an 11-year-old female patient was under the care of the paediatric intensive care unit (PICU) with severe diabetic ketoacidosis and septic shock with multiple organ failure. She required ventilation, multiple inotropes and continuous veno-venous haemofiltration and was on intravenous broad-spectrum antibiotics. The PICU team requested a dental opinion in order to rule out dental pathology as there was a history of attendance at a dental surgery with pain a week earlier. Extra-orally, the patient did not appear to have a significant facial swelling. Intraoral examination revealed multiple unrestorable carious decidious teeth. There was a buccal swelling associated with the cavitated primary upper left canine and primary upper left first molar. A decision was made to extract both these teeth under local anaesthesia in addition to her continuing sedation and muscle relaxation.

A few days following the dental extractions, the general health of the patient improved and she was discharged from PICU, and then sent home having made a full recovery.

Although it cannot be confirmed with certainty that the dental abscess was the source of the systemic infection, nonetheless, the child's health improved significantly after the removal of the carious teeth, and no other source of infection was ever clinically suspected or proven.

The patient's mother explained that her daughter had seen her general dental practitioner approximately a week earlier and extraction of the carious teeth had been advised. However, the patient was not willing to have this carried out under local anaesthesia. This case highlights the need to treat carious deciduous teeth, especially if the child has an underlying medical problem. ${ }^{1,2}$

S. Shah,

C. Mason,

J. Brierley

Great Ormond Street Hospital for Children

1. Shaw L. Prevention of dental caries in children. Int J Paed Dent 1997; 7: 268-272.

2. Fayle SA, Welbury R R, Roberts J F. Management of caries in the primary dentition. Int J Paediatr Dent 2001; 11: 153-157.

DOI: 10.1038/sj.bdj.2008.512

\section{CDTs FIND FAVOUR}

Sir, as a dental technician and a maxillofacial technologist for 38 years but now a website developer I believe that registration with a regulatory body is absolutely essential for any health care worker, regardless of patient contact. I do, however, have my reservations of how the Government has not seen the need to put in place some sort of training sooner for clinical dental technicians (CDTs) or denturists as they are sometimes known.

The Nuffield Committee recommended the introduction of CDTs in the 1990s but little has been done to create a pathway to train technicians. Many dental professionals blame the GDC for the situation but this is a bit like 'shooting the messenger'. The GDC is not there to do anything other than protect the public, perhaps sometimes in a slightly misguided way, but they are only doing their jobs to the best of their ability. I have met with many GDC members over the years and have always found them to be professional and approachable.

I am delighted that there are now about 80 or so CDTs who have registered with the GDC but at what cost? I can't begin to imagine how much some of these qualified CDTs have spent on their education but we are talking circa $£ 30,000$ just to do the George Brown Course in Toronto, not to mention major subscription charges of certain associations and loss of earnings while travelling to Canada.

What I did not realise until recently but which has become very apparent is 
how many dentists also would like to see the introduction of CDTs. This is especially so with new techniques in implantology and cosmetic dentistry which in my opinion more suits dental clinicians than prosthodontics, although there will always be a few exceptions.

N. Hancock

By email

DOI: 10.1038/sj.bdj.2008.513

\section{LEGO OF THAT}

Sir, we would like to share with you a recent amusing incident which turned up as an emergency at our practice. We were presented with a slightly worried father and a distraught 7-year-old boy. The 7-year-old boy had never attended our practice before and his father had enquired at the reception if we could see him as he had 'a piece of LEGO stuck in his tooth'. We suspected the young boy had managed to trap a slither of LEGO interproximally. However, you can understand our surprise when he opened his mouth to confirm the presence of a full size, black square LEGO block stuck firm over his lower right central incisor (Fig. 1). The LEGO piece was so firm it was too difficult to prise it off. The boy was very anxious and becoming increasingly upset so it was decided to cut it off as one would remove a temporary crown. Thankfully once the LEGO was removed the tooth below was revealed to be unharmed. I wonder if in future LEGO could modify such pieces to be used as temporary crowns. Although the

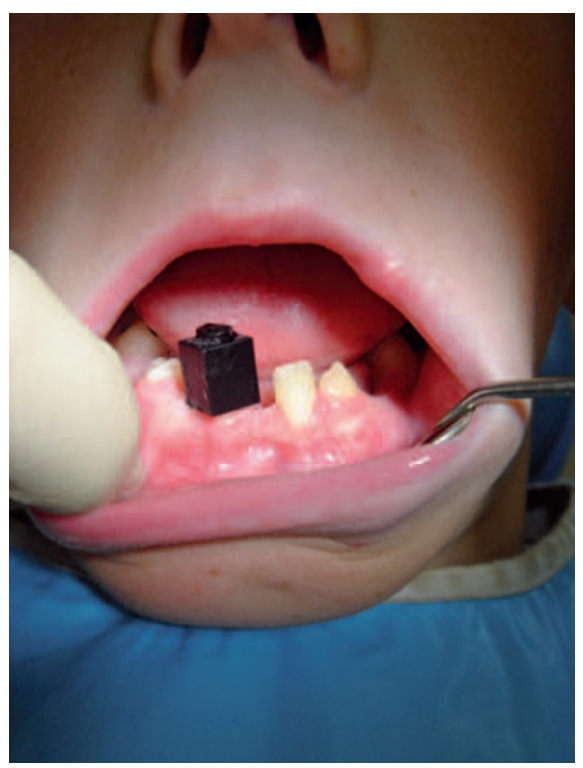

Fig. 1 LEGO block attached to incisor boy's father was relieved the LEGO was removed, his son was a little upset that he was now missing the last piece from his new LEGO spaceship!

D. C. Baker

S. D. Flaherty

St Helens

DOI: 10.1038/sj.bdj.2008.514

\section{A BACKWARD STEP}

Sir, Surrey PCT recently sent application requests from local dentists to apply for a 'Dentists with Special Interests' (DwSI) position in minor oral surgery. Having checked the FGDP criteria I felt I was well suited to this position.

When denied an interview I requested an explanation, to which Surrey PCT have stated that they want a candidate who is on the specialist list for oral surgery - that is their requirement despite the FGDP guidelines. Does this not defeat the whole point of having a DwSI in the first place? Next will I be told I need to be a DwSI prior to getting on the specialist list?

I am confused that being on the specialist list is one of the PCT's criteria when it is not one of the FGDP's criteria. It is my understanding that the General Dental Council are in the process of no longer registering dentists' additional qualifications, with a long-term view to abolish the specialists registers altogether. This is why I understood the whole concept of dentists with special interests was developed.

Enabling the general dental practitioner with postgraduate skills to be recognised and to practise these skills on the NHS public will thus benefit those with the greatest need and highest demand and reduce the strain on the hospital services. The GDC have made changes to the specialist lists; the FDGP have made recommendations and specific guidelines to benefit public need using general practitioners with additional skills. Surely this is a great concept? Yet the PCT ignore this: a backward step. Will the $B D J$ help clarify what is happening please?

\section{Bhargava By email}

Penny Stayte, BDA Head of Practice Support Service responds: First of all, we sympathise with Dr Bhargava's situation. The Dentist with a Special Interest concept was set up to cater for dentists that are not registered specialists but nevertheless have the competences required to provide care requiring additional skills and experience in primary care. Fundamentally, however, PCTs can set their own criteria for awarding contracts and where there is competition, they must be able to award the contract to the most suitable practitioners. However, if they were looking for specialists only, then it would have been normal practice to make this clear in the advertisement. I suggest anyone finding themselves in this situation contact their Local Dental Committee who can raise the issue formally with the PCT. It is also possible to make a complaint to the PCT regarding the selection process.

DOI: 10.1038/sj.bdj.2008.515

\section{SENSIBLE AND TRUE}

Sir, I thought it was very nice of Hew Mathewson, President of the GDC, to write to all of us recently (GDC Gazette spring 2008) asking us to behave better and not be nasty to each other. Some of my best friends are in private practice so I think I know what he means. It was also very helpful to be reminded that the GDC exists to protect the public from us. Everything he said was sensible and true and I think he must be a very nice man.

There is just one thing that puzzles me: repeated studies have shown (1988, 1998 Adult Dental Health Surveys) that patients who go regularly to the dentist are, in some ways, worse off than people who only go when they have a problem. Having more abscesses and having fewer sound teeth seems quite important to me. It is difficult to separate the conditions that dentists work under from what they do and so, if what we do is not very good, does that mean the conditions are not very good?

Do you think if Hew Mathewson wants to protect the public he ought to ask the Department of Health about the GDS contract? Perhaps they are partly to blame for our poor performance in the past and I can't help thinking that the next survey will show things to be worse. For example, everyone knows 
that those shiny metal dentures are more hygienic than the plastic sort but I can't see anyone making one of those now on the NHS.

N. Cole

By email

DOI: 10.1038/sj.bdj.2008.516

\section{CPD VARIETY IS GOOD}

Sir, I am writing to respond to A. Gould's letter (BDJ 2008; 204: 478), about accumulating CPD hours.

Two points are made regarding CPD certification in that it can be awarded easily even if scoring zero on CPD articles and it awards more time than it actually takes to read the article, for example awarding one hour of CPD if the task takes only ten minutes.

In my opinion, A. Gould misses the point entirely. Articles have varying levels of difficulty so that some may take 30 minutes to read and answer questions while others may take 70 minutes. In addition, some readers can read quickly and answer questions easily while other readers take longer. Another factor to consider is that some readers can assimilate certain types of information and topics far more easily than others.

CPD exists in a variety of formats and lengths with the objective of enhancing the development of the dental surgeon through added skill/knowledge or to consolidate those skills/knowledge for patient care. As CPD exists in many varieties on various dental topics there is a choice for each type of dental surgeon that learns differently.

Finally, A. Gould inconsiderately mentioned that the GDC should intervene and provide standards for CPD providers. In my opinion, it is unfair to request the GDC to intervene in this way since all providers are credible. I assume all CPD providers currently follow GDC defined criteria to award verifiable CPD hours. Setting a standard that A. Gould professes to should not relate to CPD providers, but to the subjective learning by all dentists concerned.

S. Shah

London

DOI: 10.1038/sj.bdj.2008.517

\section{CONUNDRUM CLARITY}

Sir, I should also like to thank the authors of the NICE clinical guideline 64 'Prophylaxis against infective endocarditis' for their clarity on such an important issue (Keightley A. Turned on its head. Br Dent $J$ 2008; 204: 544).

Gone is the conundrum of whether or not to give antibiotic cover for that new patient with a history of rheumatic fever who has clearly had extractions in the past without antibiotic cover with no adverse effect.

I remember as a student the obsession everyone had with the prevention of endocarditis in the 'at risk' patient. So much so that it impinges heavily on my memory of the day of my final examinations at Liverpool in 1972.

Some students were allocated a patient who had turned up in the admissions department that day for emergency treatment and we were required to diagnose their problem and if necessary treat them.

My patient had attended with toothache and wanted a tooth extracting. After taking a medical history I discovered that she had had rheumatic fever but she had no knowledge of any heart valve damage. On examination of her mouth it was clear that she had had extractions in the past without antibiotic cover.
When I presented the patient to the examiners the majority of the 'grilling' revolved around the prevention of endocarditis rather than the treatment which the patient needed. The patient at this point was becoming quite agitated listening to the sequelae that could befall her.

Because of the time restraints of the examination I never did get to extract the tooth. The patient had to go to another department for an injection of 'Triplopen' (treatment of choice in 1972 at Liverpool) and wait an hour before the tooth was extracted.

Now I will be able to carry out emergency treatment immediately for the 'at risk' patient, after of course, the necessary counselling!

$$
\begin{array}{r}
\text { K. Maunder } \\
\text { Spondon }
\end{array}
$$

\section{FAITH SENSITIVITY}

Sir, the letter by T. Sebastian ${ }^{1}$ highlights some issues reflecting faith and culture which increasingly impact upon dental professionals. This rapidly changing nature of many communities worldwide is the main reason that we encouraged the establishment of transcultural teaching on the initiative of Raman Bedi and Raj Rayan, followed by the establishment of the WHO Collaborating Centre for Oral Health, Disability and Culture (http://www.eastman.ucl.ac.uk/whocc/ activies_tor1.html). More detail about issues related to all main faiths can be found in the book Culturally sensitive oral healthcare (Scully C, Wilson N. Quintessence, 2006).

\section{Scully CBE}

1. Sebastian T. Muslims and gold. Br Dent J 2008; 204: 545.

DOI: 10.1038/sj.bdj.2008.519 